\title{
A POSITIVSTELLENSATZ FOR PROJECTIVE REAL VARIETIES
}

\author{
CLAUS SCHEIDERER
}

\begin{abstract}
Given two positive definite forms $f, g \in \mathbb{R}\left[x_{0}, \ldots, x_{n}\right]$, we prove that $f g^{N}$ is a sum of squares of forms for all sufficiently large $N \geq 0$. We generalize this result to projective $\mathbb{R}$-varieties $X$ as follows. Suppose that $X$ is reduced without one-dimensional irreducible components, and $X(\mathbb{R})$ is Zariski dense in $X$. Given everywhere positive global sections $f$ of $L^{\otimes 2}$ and $g$ of $M^{\otimes 2}$, where $L, M$ are invertible sheaves on $X$ and $M$ is ample, $f g^{N}$ is a sum of squares of sections of $L \otimes M^{\otimes N}$ for all large $N \geq 0$. In fact we prove a much more general version with semi-algebraic constraints, defined by sections of invertible sheaves. For nonsingular curves and surfaces and sufficiently regular constraints, the result remains true even if $f$ is just nonnegative. The main tools are local-global principles for sums of squares, and on the other hand an existence theorem for totally real global sections of invertible sheaves, which is the second main result of this paper. For this theorem, $X$ may be quasiprojective, but again should not have curve components. In fact, this result is false for curves in general.
\end{abstract}

\section{INTRODUCTION}

Let $f \in \mathbb{R}\left[x_{0}, \ldots, x_{n}\right]$ be a positive definite homogeneous polynomial. From Stengle's Positivstellensatz it follows that $f$ can be written as a sum of squares of quotients of forms with positive definite denominators. In 1995, Reznick [10] refined this observation by proving that one can always find such a representation where the denominators are powers of the form $g=x_{0}^{2}+\cdots+x_{n}^{2}$. In other words, there exists an integer $N \geq 0$ such that the form $f g^{N}$ is a sum of squares of forms. It was observed by the author (15] 2.1.8) that Reznick's theorem can be seen as a direct consequence of Schmüdgen's Positivstellensatz [17. As remarked in [15, this argument works in greater generality and shows, for any two non-constant positive definite forms $f$ and $g$ with $\operatorname{deg}(g) \mid \operatorname{deg}(f)$, that $f g^{2 N}$ is a sum of squares of forms for sufficiently large $N \geq 0$.

The present work resulted from an attempt to remove the degree restriction in this statement. Before we outline the results of this paper, we briefly sketch how this can be achieved. By replacing $f$ with $f_{1}=f l^{2 k}$ for suitable $k \geq 0$ and some linear form $l$, one satisfies the degree condition but looses strict positivity. As a consequence, Schmüdgen's theorem cannot be applied any more. Instead one uses the local-global criterion for sums of squares 14, combined with the fact that strictly positive elements in local rings are sums of squares [13, to get a sum of squares representation for $f_{1} g^{2 N}$ and some $N \geq 0$. By an elementary argument, any summand in such a representation is divisible by $l^{2 k}$. So one can cancel this factor and gets a representation of $f g^{2 N}$ as desired.

2010 Mathematics Subject Classification. Primary 14P05; secondary 14C20, 14N05, 13J30, $11 \mathrm{E} 25$.

Key words and phrases. Positivstellensatz, projective varieties, line bundles, totally real divisors, positive definite forms, sums of squares, preorderings, real algebraic geometry.

I am grateful to Amir Ali Ahmadi for inspiring discussions around the projective Positivstellensatz. 
To find the proper setting for generalizing this proof, one first observes that the result is genuinely projective, and not affine, in nature. Leaving technical details aside for the moment, it is quite clear what a conjectural generalization to projective $\mathbb{R}$-varieties $X$ and everywhere positive sections of line bundles should be. Analyzing the proof just outlined for $X=\mathbb{P}^{n}$, one sees that the least obvious step in proving such a generalization is to find a suitable substitute for the linear form $l$. This leads to a question of its own interest, namely totally real divisors on $\mathbb{R}$-varieties.

There are two main results in this paper. The first is an existence theorem for totally real (Cartier) divisors on quasi-projective real varieties, proved in Sect. 3 . More specifically, if $X$ is a reduced quasi-projective $\mathbb{R}$-scheme without one-dimensional irreducible component and with $X(\mathbb{R})$ Zariski dense in $X$, and if $D$ is a divisor on $X$, then for any ample divisor $E$ and any sufficiently large integer $n$ there exists a totally real (reduced) effective divisor that is linearly equivalent to $D+n E$. Here we call an effective divisor $D$ totally real if the $\mathbb{R}$-points of (the closed subscheme) $D$ are Zariski dense in $\operatorname{supp}(D)$. In fact we prove a more general version of this result which is relative to any Zariski dense semi-algebraic subset $S$ of $X(\mathbb{R})$ (Theorem 3.5). The proof is based on Bertini's first theorem. As a corollary we obtain that any divisor on $X$ is equivalent to a difference of two totally real (reduced) effective divisors, and even to a single such divisor when $X$ is affine; again we provide a more general version relative to a semi-algebraic set $S$. For projective curves, Theorem 3.5 and its corollaries fail in general, see the discussion starting in 3.10 . The result can be saved for nonsingular curves under stronger assumptions on $S$.

The second main result is a general Positivstellensatz for reduced projective $\mathbb{R}$ schemes $X$ (Theorem 4.1), proved in Sect.4. Basically it says that a strictly positive global section $f$ of an invertible sheaf $L^{\otimes 2}$ becomes a sum of squares after multiplication with a sufficiently high even power of any nowhere (on $X(\mathbb{R})$ ) vanishing section of any ample invertible sheaf. The substitute in the proof for the linear form $l$ above is a totally real section of a suitable invertible sheaf. The existence of such a section is guaranteed by Theorem 3.5. We have to assume that $X$ has no one-dimensional irreducible component since 3.5 is known to fail for curves. We do not know if the dimension restriction for the irreducible components of $X$ can be removed in Theorem 4.1. Again, the version we prove is considerably more general, and is a preordering-type statement relative to semi-algebraic constraints. For nonsingular curves and surfaces we can even prove a Nichtnegativstellensatz $(f$ is allowed to have zeros), as long as the constraints are sufficiently regular.

As a very particular concrete application it follows from Theorem 4.1 that, for any strictly positive form $f \in \mathbb{R}\left[x_{0}, \ldots, x_{n}\right]$, there exists some odd power $f^{2 m+1}$ that is a sum of squares (Corollary 4.7). Our Positivstellensatz (in the version for $\left.\mathbb{P}^{n}\right)$ was recently applied by Ahmadi and Parrilo [1] to time continuous dynamical systems.

In Sect. 2 we introduce the concept of totally real divisors and its generalization relative to a semi-algebraic set, the weakly compatible divisors. Moreover we define the sign of a section of an invertible sheaf $L^{\otimes 2}$ at a real point, and we discuss basic properties of these notions that are used later.

For proving (and even formulating) the results of this paper, it is necessary to use the language of schemes, although some particular cases can be phrased in a more naive language. In a few places we also have to work with the real spectrum. A brief discussion of the concepts used is given in a first section with preliminaries.

\section{Notations AND PRELIMINARIES}

1.1. Let $k$ be a field and $X$ a $k$-scheme of finite type. For any $k$-algebra $E$ we write $X(E)=\operatorname{Hom}_{k}(\operatorname{Spec} E, X)$ for the set of $E$-valued points of $X$. We often confuse an 
element of $X(k)$ with its closed image point in $X$. By $X_{\text {reg }}$ we denote the regular locus of $X$, i.e. the set of $x \in X$ for which the local ring $\mathcal{O}_{X, x}$ is regular; this is an open subset of $X$.

1.2. A general reference for semi-algebraic sets and the real spectrum is 3 . The real spectrum of the $\operatorname{ring} A$ is denoted $\operatorname{Sper}(A)$. Let $R$ be a real closed field, and let $X$ be a separated $R$-scheme of finite type. A subset $S$ of $X(R)$ is semi-algebraic if $S \cap U(R)$ is a semi-algebraic subset of $U(R)$ for every open affine subset $U$ of $X$. From the order topology of $R$ we get a topology on $X(R)$ that is sometimes referred to as the euclidean topology (as opposed to the Zariski topology on $X$ ). If $X$ is affine, then $X(R)$ is in the natural way a topological subspace of Sper $R[X]$. With each semi-algebraic subset $S$ of $X(R)$ one associates a constructible subset $\widetilde{S}$ of Sper $R[X]$, in such a way that $S=X(R) \cap \widetilde{S}$; in fact, $\widetilde{S}$ is defined by the same system of inequalities inside Sper $R[X]$ as $S$ inside $X(R)$. It is well-known that $\widetilde{S}$ is open (resp. closed) in Sper $R[X]$ if (and only if) the same is true for $S$ in $X(R)$. It would be natural to extend the construction of the real spectrum and of the tilde operator to non-affine $X$, but this is not needed here.

Let $R$ be a real closed field. The following is a well-known consequence of the Artin-Lang theorem. For lack of a suitable reference we give a short indication of the proof.

Proposition 1.3. Let $X$ be an integral $R$-scheme of finite type, and let $S \subseteq X(R)$ be a semi-algebraic subset. Then $S$ is Zariski dense in $X$ if and only if $S$ contains a non-empty (euclidean) open subset of $X_{\mathrm{reg}}(R)$.

Proof. One easily reduces to showing: If $X$ is affine and nonsingular, then for any $0 \neq g \in R[X]$, the zero set of $g$ in $X(R)$ has empty interior. To prove this, assume that $f_{1}, \ldots, f_{r} \in R[X]$ are such that $U:=\left\{\xi \in X(R): f_{i}(\xi)>0, i=1, \ldots, r\right\}$ is nonempty and $g \equiv 0$ on $U$. The function field $R(X)$ of $X$ has an ordering $\alpha$ which makes $f_{1}, \ldots, f_{r}$ positive. By the (refined) Artin-Lang theorem (see for instance [2] Thm. 1.3), there exists $\xi \in U$ with $\operatorname{sgn}_{\alpha}(g)=\operatorname{sgn} g(\xi)$, and in particular, $g(\xi) \neq 0$, contradiction.

Corollary 1.4. For an integral $R$-scheme $X$ of finite type, $X(R)$ is Zariski dense in $X$ if and only if $X$ has a nonsingular $R$-point, if and only if the function field $R(X)$ of $X$ is real, i.e. can be ordered.

Proof. The equivalence $X_{\text {reg }}(R) \neq \varnothing \Leftrightarrow R(X)$ real is proved in [2].

\section{Divisors AND INVERTIBLE SHEAVES}

In this section let $R$ be a real closed field, and let always $X$ be a reduced separated $R$-scheme of finite type.

2.1. By a divisor on $X$ we always mean a Cartier divisor. An effective Cartier divisor on $X$ is the same as a closed subscheme $D$ of $X$ whose sheaf of ideals is everywhere locally generated by one element that is not a zero divisor. The support $\operatorname{supp}(D)$ of $D$ is the closed subset of $X$ underlying $D$.

Let $D$ be an effective divisor on $X$, and let $S \subseteq X(R)$ be a semi-algebraic set. We say that $D$ is weakly compatible with $S$ if $S \cap D(R)$ is Zariski dense in $\operatorname{supp}(D)$. We call $D$ totally real if $D$ is weakly compatible with $S=X(R)$.

Remark 2.2. In [8, the notion of compatibility between a semi-algebraic set $S \subseteq$ $X(R)$ and a prime Weil divisor $Y$ on $X$ was introduced (in the case where $X$ is normal). The notion from [8] is stronger (at least when $S$ is closed) than weak compatibility as defined in 2.1, which explains our choice of terminology here. The 
existence results for weakly compatible sections proved in Sect. 3 below remain true for the stronger notion of compatibility (suitably adapted to the more general situation when $X$ is not normal). However, that notion is more technical and is not needed here, which is why we work with the easier concept of weak compatibility.

2.3. Let $L$ be an invertible sheaf (locally free $\mathcal{O}_{X}$-module of rank one) on $X$. Given a global section $s$ of $L$, let $Z(s)$ be the closed zero subscheme of $s$. The section $s$ is said to be regular if $Z(s)$ is an effective divisor on $X$. Since $X$ is reduced, it is equivalent that $\operatorname{supp}(s)$ does not contain any irreducible component of $X$. A regular section $s$ of $L$ is called reduced if the closed subscheme $Z(s)$ of $X$ is reduced. We say that the regular section $s$ is weakly compatible with $S$ if the effective divisor $Z(s)$ is weakly compatible with $S$, c.f. Definition 2.1. Again, we call $s$ totally real if $s$ is weakly compatible with $S=X(R)$.

If $L, M$ are invertible sheaves and $s$ resp. $t$ are global sections of $L$ resp. $M$, we denote by $s t$ the product $s \otimes t$, seen as a global section of $L \otimes M$.

2.4. Let $L, L^{\prime}$ be invertible sheaves on $X$, and let $\phi: L \otimes L \stackrel{\sim}{\rightarrow} L^{\prime}$ be an isomorphism. Fixing $\phi$, we can talk of the sign of global (or local) sections of $L^{\prime}$ at points $\xi \in X(R)$. Namely, given $f \in H^{0}\left(X, L^{\prime}\right)$ and $\xi \in X(R)$, the sign of $f$ at $\xi$ (with respect to $\phi$ ) is defined as

$$
\operatorname{sgn}_{\xi, \phi}(f):=\operatorname{sgn}_{\xi}(a) \in\{-1,0,1\},
$$

where $U \subseteq X$ is an open set with $\xi \in U(R)$ over which $L$ is trivial, $s \in H^{0}(U, L)$ is a generator of $\left.L\right|_{U}$ and $a \in \mathcal{O}_{X}(U)$ is defined by $\left.f\right|_{U}=a \cdot \phi\left(s^{2}\right)$. We say that $f$ is nonnegative, resp. (strictly) positive (with respect to $\phi$ ), if $\operatorname{sgn}_{\xi, \phi}(f) \geq 0$, resp. $>0$, for every $\xi \in X(R)$. When $\phi$ is understood, we simply write $f(\xi) \geq 0$ or $f(\xi)>0$, instead of $\operatorname{sgn}_{\xi, \phi}(f) \geq 0$ or $\operatorname{sgn}_{\xi, \phi}(f)=1$, respectively.

It is clear that the definition of $\operatorname{sgn}_{\xi, \phi}(f)$ does not depend on the choice of either $U$ or $s$. It does depend, however, on the square root $L$ of $L^{\prime}$ and on the choice of the isomorphism $\phi$.

\section{Remarks 2.5.}

1. Throughout this paper, we will only talk of signs of sections of invertible sheaves $L^{\prime}$ that are given in the form $L^{\prime}=L \otimes L$. These signs are always understood with respect ot the identity isomorphism of $L \otimes L$. Therefore, we will consequently suppress mentioning the isomorphism $\phi$. For example, if $s \in H^{0}\left(X, L^{\otimes 2}\right)$ and $t \in H^{0}\left(X, M^{\otimes 2}\right)$, then for the signs of $s t \in H^{0}\left(X,(L \otimes M)^{\otimes 2}\right)$ we have $\operatorname{sgn}_{\xi}(s t)=$ $\operatorname{sgn}_{\xi}(s) \cdot \operatorname{sgn}_{\xi}(t)$, for $\xi \in X(R)$.

2. Recall that $X$ denotes a (reduced separated) $R$-scheme of finite type. If $L$ is an invertible sheaf on $X$ and $f \in H^{0}\left(X, L^{\otimes 2}\right)$, then $S=\{\xi \in X(R): f(\xi) \geq 0\}$ is a closed semi-algebraic subset of $X(R)$.

Definition 2.6. Let $L, M$ be invertible sheaves on a scheme $X$, and let $s$ resp. $t$ be global sections of $L$ resp. $M$. We say that $s$ divides $t$, if there is a global section $u$ of $L^{\vee} \otimes M$ with $t=s u$.

The following lemma is clear:

Lemma 2.7. Let $L, M$ be invertible sheaves on $X$, let $s \in H^{0}(X, L)$ and $t \in$ $H^{0}(X, M)$.

(a) $s$ divides $t$ if and only if $X$ has a covering by open subsets $U_{\alpha}$ such that $\left.s\right|_{U_{\alpha}}$ divides $\left.t\right|_{U_{\alpha}}$ for all $\alpha$.

(b) If $s$ is regular, and if $t^{\prime} \in H^{0}(X, M)$ satisfies st $=s t^{\prime}$ in $H^{0}(X, L \otimes M)$, then $t=t^{\prime}$. 
Lemma 2.8. Let $L, M, N$ be invertible sheaves on $X$ with regular global sections $s, t, u$, respectively, and assume that $s$ divides $t u$. If $Z(s)$ is reduced, and if $t$ does not vanish identically on any irreducible component of $\operatorname{supp}(s)$, then $s$ divides $u$.

Proof. We can localize und assume that $s, t, u$ are not zero divisors in the $\operatorname{ring} A$, and that $(s)=\sqrt{(s)}$. If $\mathfrak{p}_{1}, \ldots, \mathfrak{p}_{r}$ are the minimal prime ideals containing $s$, then the hypothesis says $t \notin \mathfrak{p}_{1} \cup \cdots \cup \mathfrak{p}_{r}$. Since $t u \in(s)$ by hypothesis, we conclude $u \in \bigcap_{i} \mathfrak{p}_{i}=(s)$.

The following lemma is the technical reason why weak compatibility is a key notion for this paper:

Lemma 2.9. Let $X$ be an $R$-scheme of finite type, and let $S \subseteq X(R)$ be a semialgebraic set. Let $L, M$ be invertible sheaves on $X$, and let $s \in H^{0}(X, L)$ be a regular and reduced global section that is weakly compatible with $S$.

(a) If $a, b \in H^{0}\left(X, M^{\otimes 2}\right)$ are nonnegative on $S$, and if $s$ divides $a+b$, then $s$ divides both $a$ and $b$.

(b) If $a_{1}, \ldots, a_{r} \in H^{0}(X, M)$ and $s$ divides $a_{1}^{2}+\cdots+a_{r}^{2}$, then $s$ divides $a_{1}, \ldots, a_{r}$.

Proof. We may argue locally. Let $x$ be a closed point of $X$ and let $A=\mathcal{O}_{X, x}$ be the local ring of $X$ at $x$. By fixing trivializations of $L$ and $M$ at $x$ we may identify the values of local sections of these sheaves at the stalk $x$ with elements of $A$. Let $f \in A$ correspond to $s$. The assumption on $s$ means that $(f)=\mathfrak{p}_{1} \cap \cdots \cap \mathfrak{p}_{r}$ with prime ideals $\mathfrak{p}_{i}$ of $A$, and that for $i=1, \ldots, r$ there exists $\alpha_{i} \in \widetilde{S} \cap \operatorname{Sper}(A)$ with $\operatorname{supp}\left(\alpha_{i}\right)=\mathfrak{p}_{i}$.

For (a) let $g, h \in A$ correspond to $a$ resp. $b$. By assumption, $a$ and $b$ are nonnegative on $\widetilde{S}$. For every $i=1, \ldots, r$ we have $g+h \in \mathfrak{p}_{i}$, that is, $(g+h)\left(\alpha_{i}\right)=0$, and on the other hand $g\left(\alpha_{i}\right) \geq 0$ and $h\left(\alpha_{i}\right) \geq 0$. Together this implies $g, h \in \mathfrak{p}_{i}$, and hence $g, h \in(f)$. For (b) let $g_{j} \in A$ correspond to $a_{j}$. From (a) it follows that $s$ divides $a_{j}^{2}$, whencee $g_{j}^{2} \in(f)$, for every $j$. Since $(f)=\sqrt{(f)}$ we conclude $g_{j} \in(f)$.

\section{Existence of totally REAL SECTIONS}

The main result of this section is Theorem 3.5. which is an existence theorem for totally real global sections of invertible sheaves on quasi-projective real varieties. Throughout this section let $R$ denote a real closed base field.

The following lemma is an application of the (semi-algebraic) theorem on implicit functions:

Lemma 3.1. Let $X$ be a reduced $R$-scheme of finite type and let $f: X \rightarrow \mathbb{A}^{m}$ be an $R$-morphism. For $0 \neq a \in R^{m}$ let $X_{a} \subseteq X$ be the (scheme-theoretic) preimage under $f$ of the linear hyperplane $\sum_{i=1}^{m} a_{i} x_{i}=0$ in $\mathbb{A}^{m}$. Let $0 \neq a \in R^{m}$ be such that $X_{a}$ contains no irreducible component of $X$, and assume that $X_{a}$ has a regular $R$ point $\xi$. Given an open neighborhood $U$ of $\xi$ in $X(R)$, we have $U \cap\left(X_{b}\right)_{\mathrm{reg}}(R) \neq \varnothing$ for all $b \in R^{m}$ close to $a$.

Proof. Let $W \subseteq X \times \mathbb{A}^{m}$ be the closed subscheme whose $E$-valued points are the pairs $(\eta, b) \in X(E) \times E^{m}$ with $\sum_{i} b_{i} f_{i}(\eta)=0$, for $E$ an $R$-algebra. The point $\xi$ is a regular point of $X$, and the tangent space to $W$ at $(\xi, a)$ consists of the pairs $(u, v) \in T_{\xi}(X) \oplus R^{m}$ for which $\left\langle d_{\xi} f_{a}, u\right\rangle+\langle f(\xi), v\rangle=0$. Here $d_{\xi} f_{a} \in T_{\xi}(X)^{*}$ denotes the differential of $f_{a}:=\sum_{i} a_{i} f_{i}$ at $\xi$. Since $\xi$ is a regular point of $X_{a}$ (and $X_{a}$ doesn't contain a Zariski neighborhood of $\xi$ ) we have $d_{\xi} f_{a} \neq 0$, and so $(\xi, a)$ is a regular point of $W$. The projection $\pi: W \rightarrow \mathbb{A}^{m}$ is submersive at $(\xi, a)$. By the (semi-algebraic) theorem on implicit functions (3] Cor. 2.9.8) there is a local continuous (semi-algebraic) section $b \mapsto(\eta(b), b)$ of $\pi$ around $a$ with $\eta(a)=\xi$. So 
$\eta(b)$ is an $R$-point of $X_{b}$ and is, by continuity, a regular point of $X_{b}$, for $b$ close to $a$.

Lemma 3.2. Let $X$ be a reduced $R$-scheme of finite type, let $L$ be an invertible sheaf on $X$, and let $V \subseteq H^{0}(X, L)$ be a finite-dimensional linear subspace. Moreover let open semi-algebraic subsets $U_{1}, \ldots, U_{r}$ of $X(R)$ be given. Then the set of all regular sections $s \in V$ with $Z(s)_{\mathrm{reg}} \cap U_{i} \neq \varnothing$ for $i=1, \ldots, r$ is open in $V$ (in the euclidean topology).

Proof. Being a regular global section is an open condition on $s$. Given $s \in V$ with $Z(s)_{\text {reg }} \cap U_{i} \neq \varnothing$ for $i=1, \ldots, r$, it follows from Lemma 3.1 that any $t \in V$ sufficiently close to $s$ satisfies $Z(t)_{\text {reg }} \cap U_{i} \neq \varnothing$ for $i=1, \ldots, r$ as well.

Lemma 3.3. Let $k$ be a field and let $X$ be a reduced quasi-projective $k$-scheme without 0-dimensional irreducible components. Let $L, M$ be invertible sheaves on $X$, with $M$ ample. Let finitely many closed points $\xi_{1}, \ldots, \xi_{r}$ and $\eta_{1}, \ldots, \eta_{p}$ in $X$ be given, with $\xi_{i} \neq \eta_{j}$ for all $i, j$, and assume that $\xi_{1}, \ldots, \xi_{r}$ are regular points of $X$. There is an integer $n_{0} \geq 0$ such that, for any $n \geq n_{0}$, the sheaf $L \otimes M^{\otimes n}$ has a regular global section $s$ for which $\xi_{1}, \ldots, \xi_{r}$ are regular points of $Z(s)$ and $\eta_{1}, \ldots, \eta_{p} \notin Z(s)$.

Proof. Assume the lemma is shown in the case where $M$ is very ample. In the general case there is $d \geq 1$ such that $M^{\otimes d}$ is very ample. For $i=1, \ldots, d$ there exists, by the assumption, an integer $n_{i} \in \mathbb{N}$ such that, for $n \geq n_{i}, L \otimes M^{\otimes(i+d n)}$ has a regular section as required. Therefore, if $n \geq \max \left\{i+d n_{i}: i=1, \ldots, d\right\}$, then $L \otimes M^{\otimes n}$ has a regular section as required.

So we may assume that $X \subseteq \mathbb{P}^{m}$ is locally closed and $M=\mathcal{O}_{X}(1)$. Let $\bar{X} \subseteq \mathbb{P}^{m}$ be the reduced closure of $X$. There exists a coherent sheaf $L^{\prime}$ on $\bar{X}$ for which $\left.L^{\prime}\right|_{X} \cong L$ ([4] exercise II.5.15). By adding more points to the sequence $\eta_{1}, \ldots, \eta_{p}$ if necessary we may assume that every irreducible component of $X$ contains one of the $\eta_{j}$. Consider the 0 -dimensional closed subscheme

$$
Y:=\coprod_{i=1}^{r} \operatorname{Spec}\left(\mathcal{O}_{X, \xi_{i}} / \mathfrak{m}_{X, \xi_{i}}^{2}\right) \amalg \coprod_{j=1}^{p} \operatorname{Spec} k\left(\eta_{j}\right)
$$

of $X$, where $k\left(\eta_{j}\right)=\mathcal{O}_{X, \eta_{j}} / \mathfrak{m}_{X, \eta_{j}}$ denotes the residue field of $\eta_{j}$, and let $i: Y \hookrightarrow \bar{X}$ be the inclusion. We have the exact sequence

$$
0 \rightarrow F \rightarrow L^{\prime} \rightarrow i_{*} i^{*} L^{\prime} \rightarrow 0
$$

of coherent sheaves on $\bar{X}$. There exists $n_{0} \geq 0$ such that $H^{1}(\bar{X}, F(n))=0$ for all $n \geq n_{0}$ ([4] III.5.2). Hence the restriction map

$$
H^{0}\left(\bar{X}, L^{\prime}(n)\right) \rightarrow H^{0}\left(Y, L^{\prime}(n)\right)
$$

is surjective for $n \geq n_{0}$. In particular, for every $n \geq n_{0}$, there is a section $s \in$ $H^{0}\left(\bar{X}, L^{\prime}(n)\right)$ that vanishes in each of $\xi_{1}, \ldots, \xi_{r}$, but only of first order, and that does not vanish in any of $\eta_{1}, \ldots, \eta_{p}$. It is clear that $\left.s\right|_{X}$ is a regular global section of $L(n)$, and that $\xi_{1}, \ldots, \xi_{r}$ are nonsingular points of $Z\left(\left.s\right|_{X}\right)$.

We recall the following consequences of Bertini's theorems.

Proposition 3.4. Let $k$ be a field of characteristic zero, and let $X$ be a reduced and locally closed $k$-subscheme of $\mathbb{P}^{n}$.

(a) For almost all $k$-hyperplanes $H$ the intersection $H \cap X$ is reduced.

(b) If $X$ is geometrically irreducible and $\operatorname{dim}(X) \geq 2$, then for almost all $k$ hyperplanes $H$ the intersection $H \cap X$ is irreducible. 
Here, as usual, "for almost all $k$-hyperplanes $H$ " means: For all $k$-hyperplanes that lie in a non-empty open subset of the dual projective space $\left(\mathbb{P}_{k}^{n}\right)^{*}$.

Proof. See [5] Cor. 6.11.

Theorem 3.5. Let $R$ be a real closed field and $X$ a reduced quasi-projective $R$ scheme with $\operatorname{dim}\left(X^{\prime}\right) \geq 2$ for every irreducible component $X^{\prime}$ of $X$. Let $S \subseteq X(R)$ be a semi-algebraic set that is Zariski dense in $X$.

(a) If $L, M$ are invertible sheaves on $X$ and $M$ is ample, there exists $n_{0} \geq 0$ such that, for every $n \geq n_{0}, L \otimes M^{\otimes n}$ has a regular and reduced global section $s$ that is weakly compatible with $S$.

(b) If $X$ is irreducible, any very ample invertible sheaf on $X$ has a nonzero reduced global section that is weakly compatible with $S$.

Proof. (a) As in the proof of Lemma 3.3. we can assume that $M$ is very ample. So we may assume that $X \subseteq \mathbb{P}^{n}$ is locally closed and $M=\mathcal{O}_{X}(1)$. Let $X_{1}, \ldots, X_{r}$ be the irreducible components of $X$. Since $S$ is Zariski dense in $X$, there exists. for each $i=$ $1, \ldots, r$, a non-empty open set $U_{i} \subseteq X_{\text {reg }}(R) \cap X_{i}(R)$ contained in $S$ (Proposition 1.3). For $i=1, \ldots, r$ fix a point $\xi_{i} \in U_{i}$. Choose $n_{0} \geq 0$ so large that the conclusion of Lemma 3.3 holds for $\xi_{1}, \ldots, \xi_{r}$, and that $L(n)$ is very ample for all $n \geq n_{0}$. Fix $n \geq n_{0}$ and a regular section $s \in H^{0}(X, L(n))$ for which $\xi_{1}, \ldots, \xi_{r}$ are regular points of $Z(s)$. Choose a finite-dimensional linear subspace $V \subseteq H^{0}(X, L(n))$ containing $s$ such that the pair $(L(n), V)$ defines a locally closed embedding $X \hookrightarrow \mathbb{P}^{\operatorname{dim}(V)-1}$. Inside $V$ there is an open neighborhood $\Omega$ of $s$ (with respect to the euclidean topology) consisting of regular sections $t$ satisfying $Z(t)_{\mathrm{reg}} \cap U_{i} \neq \varnothing$ for $i=1, \ldots, r$, by Lemma 3.2. On the other hand, for generic $t \in V$, Bertini's theorem 3.4 tells us that the scheme $Z(t)$ is reduced and $Z(t) \cap X_{i}$ is irreducible for $i=1, \ldots, r$. Indeed, $\operatorname{dim}\left(X_{i}\right) \geq 2$ by assumption, and $X_{i}$ is geometrically irreducible since $X_{i}$ has a regular $R$-point. In particular, there exists such $t$ in $\Omega$. For any such $t$, the reduced closed subscheme $Z(t)$ of $X$ has precisely $r$ different irreducible components $Z_{1}, \ldots, Z_{r}$, for which $Z_{i} \subseteq X_{i}$ and $\left(Z_{i}\right)_{\mathrm{reg}} \cap U_{i} \neq \varnothing(i=1, \ldots, r)$. Hence $Z_{i}(R) \cap S$ is Zariski dense in $Z_{i}$ for each $i$ (see 1.3), and so $t$ is weakly compatible with $S$ (see 2.1).

(b) We can assume $X \subseteq \mathbb{P}^{n}$ and $L=\mathcal{O}_{X}(1)$. There is a non-empty open subset $U$ of $X_{\text {reg }}(R)$ contained in $S$. Any linear hyperplane $H$ that intersects $X$ transversely in some point of $U$ and for which $H \cap X$ is reduced and irreducible corresponds to a section of $L$ that is weakly compatible with $S$.

Remark 3.6. If $X$ is reducible in Theorem 3.5, statement (b) usually fails. As an illustration let $X=X_{0} \cup \cdots \cup X_{n}$ be a union of isotropic quadrics $X_{i}$ in $\mathbb{P}_{R}^{n}$ with signature $(n, 1)$ such that the sets $X_{i}(R)$ are pairwise disjoint and not nested. If the diameters of these quadrics are sufficiently small, there is no hyperplane meeting each $X_{i}$ in a real point.

Remark 3.7. In both parts of Theorem 3.5, we may require in addition that $s$ does not vanish in a finite list of given closed points of $X$. (See Lemma 3.2.)

Corollary 3.8. Let $X$ be a reduced quasi-projective $R$-scheme with $\operatorname{dim}\left(X^{\prime}\right) \neq 1$ for every irreducible component $X^{\prime}$ of $X$, and let $S \subseteq X(R)$ be a semi-algebraic subset that is Zariski dense in $X$.

(a) Any divisor on $X$ is linearly equivalent to a difference $D_{1}-D_{2}$, where $D_{1}$, $D_{2}$ are reduced effective divisors that are weakly compatible with $S$.

(b) If $X$ is affine, any divisor is linearly equivalent to an effective reduced divisor that is weakly compatible with $S$. 
Proof. Let $D$ be a divisor with associated invertible sheaf $\mathcal{O}_{X}(D)$, and choose a very ample invertible sheaf $M$ on $X$. If $n>0$ is large enough then $M^{\otimes n}$ and $\mathcal{O}_{X}(D) \otimes M^{\otimes n}$ have regular and reduced global sections $s$ resp. $t$ that are weakly compatible with $S$ (Theorem 3.5). Hence $D$ is linearly equivalent to $Z(t)-Z(s)$. If $X$ is affine we can take $M=\mathcal{O}_{X}$ and $s=1$.

Remark 3.9. Particular cases of Corollary 3.8 were proved by Roggero [1]. For $R=\mathbb{R}$ and $S=X(\mathbb{R})$, she proved the case where $X$ is normal of dimension $\geq 2$, and either affine or projective.

Remark 3.10. The exclusion of one-dimensional irreducible components in these results cannot be avoided. More precisely, the following is known. Let $X$ be a connected nonsingular projective curve over $R$ with $X(R) \neq \varnothing$. There exists an integer $n \geq 0$ such that any divisor $D$ on $X$ with $\operatorname{deg}(D)>n$ is linearly equivalent to an effective totally real divisor ([12] Cor. 2.10; see also Monnier [6] Thm. 3.6 who proves $n \leq 2 g-1$ for $M$-curves or $(M-1)$-curves over $\mathbb{R})$. More generally, we have, for $R=\mathbb{R}$ :

Proposition 3.11. Let $X$ be a connected nonsingular projective curve over $\mathbb{R}$, and let $S \subseteq X(\mathbb{R})$ be an infinite semi-algebraic subset with $S \cap O \neq \varnothing$ for every oval $O$ of $X(\mathbb{R})$. There is an integer $n \geq 0$ such that every invertible sheaf $L$ on $X$ with $\operatorname{deg}(L) \geq n$ has a nonzero global section that is weakly compatible with $S$.

Proof. Let $O_{1}, \ldots, O_{r}$ be the different ovals of $X(\mathbb{R})$. For $i=1, \ldots, r$, fix a point $Q_{i} \in S \cap O_{i}$. Let $J$ be the Jacobian of $X$, and let $J(\mathbb{R})_{0}$ be the identity component of the real Lie group $J(\mathbb{R})$. The class of a Weil divisor $D=\sum_{P} n_{P} P$ of degree zero on $X$ lies in $J(\mathbb{R})_{0}$ if and only if, for any oval $O$ of $X(\mathbb{R})$, the sum $\sum_{P \in O} n_{P}$ is even ([12] Lemma 2.6). By 12 Lemma 2.12 there is an integer $m \geq 1$ such that, for every $\alpha \in J(\mathbb{R})_{0}$, there exist $m$ points $P_{1}, \ldots, P_{m} \in S$ with $\alpha=\sum_{i=1}^{m}\left[P_{i}-Q_{1}\right]$. We claim that the assertion of the proposition is satisfied with $n:=m+r-1$. Indeed, let $D$ be a Weil divisor with $\operatorname{deg}(D)=d \geq m+r-1$. There exist (unique) numbers $a_{2}, \ldots, a_{r} \in\{0,1\}$ such that the class of $D-d Q_{1}-\sum_{i=2}^{r} a_{i}\left(Q_{i}-Q_{1}\right)$ lies in $J(\mathbb{R})_{0}$. Hence there exist $P_{1}, \ldots, P_{m} \in S$ such that this divisor is equivalent to $\sum_{j=1}^{m}\left[P_{j}-Q_{1}\right]$. Altogether we conclude

$$
D \sim\left(d-m-\sum_{i=2}^{r} a_{i}\right) Q_{1}+\sum_{i=2}^{r} a_{i} Q_{i}+\sum_{j=1}^{m} P_{j} .
$$

\section{Remarks 3.12.}

1. If $S$ fails to meet one of the ovals, the assertion of Proposition 3.11 clearly becomes false. If $R$ is a non-archimedean real closed field, 3.11 may also fail when $S$ intersects each oval in an open set, c.f. [12] Rem. 2.15.

2. Part (b) of Theorem 3.5 clearly fails in general, even for $R=\mathbb{R}$ and $S=X(\mathbb{R})$.

3. Now let $X / R$ be a singular integral projective curve with $|X(R)|=\infty$. If the singularities of $X$ are real nodes (possibly with nonreal tangents), Monnier [7] has proved (for $R=\mathbb{R}$ ) the existence of an integer $n \geq 0$ such that every divisor of degree $\geq n$ is linearly equivalent to some totally real divisor supported by regular points. On the other hand, there are singular curves with ample divisors no multiple of which is equivalent to a totally real divisor, as the next proposition shows.

Proposition 3.13. Let $X$ be an integral projective curve over $R=\mathbb{R}$ with $X(\mathbb{R}) \neq$ $\varnothing$. Assume that $X$ is singular, but all real points on $X$ are regular. Then for any 
$d \geq 1$ there exists an invertible sheaf $L$ on $X$ of degree $d$ such that, for any $n \geq 1$, $L^{\otimes n}$ does not have any nonzero totally real global section.

Proof. Let $J$ be the generalized Jacobian of $X$. From the assumption it follows that the real Lie group $J(\mathbb{R})$ is not compact. Fix a point $P_{0} \in X(\mathbb{R})$, and consider the map $\varphi: X(\mathbb{R}) \rightarrow J(\mathbb{R}), \varphi(P)=\left[P-P_{0}\right]$, the class of the locally principal Weil divisor $P-P_{0}$. ( $\varphi$ extends to a morphism of varieties, but we only need that $\varphi$ is a continuous map.) The image of $\varphi$ is a compact subset of $J(\mathbb{R})$. Fix $d \geq 1$, and consider the set $T R_{d}$ of all divisors $D$ of degree $d$ for which there exists $n \geq 1$ such that $n D$ is equivalent to an effective totally real divisor. We show that the subset

$$
\left\{\left[D-d P_{0}\right]: D \in T R_{d}\right\}
$$

of $J(\mathbb{R})$ is compact, thereby proving the proposition. Let $D \in T R_{d}$. There are $n \geq 1$ and $P_{1}, \ldots, P_{n d} \in X(\mathbb{R})$ with $n[D]=\sum_{i=1}^{n d}\left[P_{i}\right]$, and hence $n\left[D-d P_{0}\right]=$ $\sum_{i=1}^{n d} \varphi\left(P_{i}\right)$. Let $M_{d} \subseteq J(\mathbb{R})$ denote the set of $d$-fold sums of elements of $\operatorname{im}(\varphi)$. Then $M_{d}$ is a compact set, and $n\left[D-d P_{0}\right]$ is a sum of $n$ elements of $M_{d}$. Therefore the claim follows from the next lemma.

Lemma 3.14. Let $(G,+)$ be a commutative real Lie group with finitely many connected components. Given a subset $M$ of $G$, let

$$
C=\left\{x \in G: \exists n \geq 1 \exists y_{1}, \ldots, y_{n} \in M n x=y_{1}+\cdots+y_{n}\right\} .
$$

If $\bar{M}$ is compact, then $\bar{C}$ is compact as well.

Proof. $G$ is isomorphic to a direct product $K \times V$ where $K$ is a compact Lie group and $V$ is a real vector space of finite dimension. It suffices to prove that $\bar{C}+K$ is compact, so we may assume $G=V=\mathbb{R}^{n}$. But then $C$ is contained in the convex hull of $M$, and so the assertion is clear.

3.15. As for affine curves, we only make the following remark. Let $X$ be a nonsingular affine curve over $\mathbb{R}$, and let $S \subseteq X(\mathbb{R})$ be a non-empty open semi-algebraic subset that intersects every connected component of $X(\mathbb{R})$. Then every invertible sheaf on $X$ has a nonzero global section that is weakly compatible with $S$. Indeed, let $X \subseteq \bar{X}$ be the nonsingular completion of $X$. Let $L$ be an invertible sheaf on $X$, and choose an invertible sheaf $L_{1}$ on $\bar{X}$ with $\left.L_{1}\right|_{X} \cong L$. By Riemann-Roch, there exists an ample invertible sheaf $M$ on $\bar{X}$ such that $\left.M\right|_{X}$ is trivial. By Proposition 3.11 $L_{1} \otimes M^{\otimes n}$ has a section on $\bar{X}$ that is weakly compatible with $S$, for some $n \geq 0$. Hence $L$ has such a section on $X$.

\section{Projective Positivstellensatz}

In the following it is essential that the real closed base field is $\mathbb{R}$, the usual real numbers (or a real closed subfield thereof). The main result is:

Theorem 4.1. Let $X$ be a reduced projective $\mathbb{R}$-scheme without one-dimensional irreducible components. Let $L, M, N_{1}, \ldots, N_{r}$ be invertible sheaves on $X$, with $M$ ample, and let regular global sections $h_{i} \in H^{0}\left(X, N_{i}^{\otimes 2}\right)(i=1, \ldots, r)$ be given such that the semi-algebraic subset

$$
K=\left\{\xi \in X(\mathbb{R}): h_{1}(\xi) \geq 0, \ldots, h_{r}(\xi) \geq 0\right\}
$$

of $X(\mathbb{R})$ is Zariski dense in $X$. Let moreover $f \in H^{0}\left(X, L^{\otimes 2}\right)$ and $g \in H^{0}\left(X, M^{\otimes 2}\right)$ be given with $f(\xi)>0$ and $g(\xi)>0$ for every $\xi \in K$. Then there is $n_{0} \geq 0$ such that, for all $n \geq n_{0}$, there exist sums of squares $s_{e}$ with

$$
f g^{n}=\sum_{e \in\{0,1\}^{r}} s_{e} \cdot h_{1}^{e_{1}} \cdots h_{r}^{e_{r}}
$$


in $H^{0}\left(X, L^{\otimes 2} \otimes M^{\otimes 2 n}\right)$. (More precisely, $s_{e}$ is a sum of squares of elements of $H^{0}\left(X, F_{e}\right)$ with $F_{e}=L \otimes M^{\otimes n} \otimes \bigotimes_{i} N_{i}^{-e_{i}}$, for $e \in\{0,1\}^{r}$.)

See convention 2.5, 1 for the meaning of signs of sections at points $\xi \in X(\mathbb{R})$.

Proof. Under the assumptions of the theorem, we will prove the following apparently weaker statement: Given $f^{\prime} \in H^{0}\left(X, L^{\otimes 2}\right)$ and $g^{\prime} \in H^{0}\left(X, M^{\prime}\right)$, where $M^{\prime}$ is ample and $f^{\prime}(\xi)>0, g^{\prime}(\xi) \neq 0$ for all $\xi \in K$, there exists $n \geq 0$ such that $f^{\prime} g^{\prime 2 n}$ allows an identity (1). Put $M^{\prime}=M^{\otimes 2}$ and apply this weaker statement to $\left(f^{\prime}, g^{\prime}\right)=(f, g)$ and $\left(f^{\prime}, g^{\prime}\right)=(f g, g)$, to get Theorem 4.1.

Resetting notation, assume for the rest of this proof that $g \in H^{0}(X, M)$ vanishes nowhere on $K$. We have to show that there is an identity (11) with some even number $n \geq 0$. We can immediately dispense with 0 -dimensional irreducible components of $X$, and can therefore assume $\operatorname{dim}\left(X^{\prime}\right) \geq 2$ for every irreducible component $X^{\prime}$ of $X$. Since we can replace $M$ by $M^{\otimes m}$ (and $g$ by $g^{m}$ ) for $m \geq 1$, we may assume that $M$ is very ample. So let $X \subseteq \mathbb{P}^{n}$ be a closed subscheme, and let $M=\mathcal{O}_{X}(1)$. We consider the open affine subscheme $Y=X_{g}=\{x \in X: g(x) \neq 0\}$ of $X$. The ring $\mathbb{R}[Y]=H^{0}\left(Y, \mathcal{O}_{X}\right)$ of regular functions on $Y$ is the homogeneous localization of the graded ring $S=\bigoplus_{k \geq 0} H^{0}\left(X, \mathcal{O}_{X}(k)\right)$ by the element $g \in S_{1}$. In other words, the elements of $\mathbb{R}[Y]$ are the fractions $\frac{a}{g^{k}}$ with $k \geq 0$ and $a \in \Gamma\left(X, \mathcal{O}_{X}(k)\right)$, with the usual rules for such fractions. By the hypothesis on $g$ we have $K \subseteq Y(\mathbb{R})$. Note that $K$ is closed in $X(\mathbb{R})(2.5,2)$, therefore $K$ is a compact semi-algebraic subset of $Y(\mathbb{R})$.

For any invertible sheaf $P$ on $X$ that is generated by its global sections, there exists $s \in H^{0}\left(X, P^{\otimes 2}\right)$ with $s(\xi)>0$ for all $\xi \in X(\mathbb{R})$. Indeed, if $P$ is generated by its global sections $s_{1}, \ldots, s_{m}$, then $s:=s_{1}^{2}+\cdots+s_{m}^{2}$ has this property.

By Theorem 3.5 (a) there exists an integer $t \geq 0$ such that the invertible sheaf $L^{\vee}(t)$ on $X$ has a regular and reduced global section $h$ that is weakly compatible with $K$. By Remark 3.7, we can get in addition that $h$ does not vanish identically on any irreducible component of $\operatorname{supp}\left(h_{i}\right)$, for any $i \in\{1, \ldots, r\}$. Since the $h_{i}$ are regular, this means that also conversely no $h_{i}$ vanishes identically on any irreducible component of $\operatorname{supp}(h)$. Consider

$$
\varphi:=\frac{f h^{2}}{g^{2 t}} \in \mathbb{R}[Y]
$$

a regular function on $Y$ that is nonnegative on $K$.

For each $i=1, \ldots, r$ we choose an integer $a_{i} \geq 0$ such that the sheaf $N_{i}^{\prime}:=$ $N_{i}^{\vee}\left(a_{i}\right)$ is generated by global sections, and we choose $p_{i} \in H^{0}\left(X, N_{i}^{\prime \otimes 2}\right)$ such that $p_{i}>0$ on $X(\mathbb{R})$. Then

$$
H_{i}:=\frac{h_{i} p_{i}}{g^{2 a_{i}}} \in \mathbb{R}[Y]
$$

and

$$
K=\left\{\xi \in Y(\mathbb{R}): H_{1}(\xi) \geq 0, \ldots, H_{r}(\xi) \geq 0\right\} .
$$

In particular, the compact subset $K$ of $Y(\mathbb{R})$ is basic closed.

We shall prove that $\varphi$ lies in the preordering generated by $H_{1}, \ldots, H_{r}$ in the ring $\mathbb{R}[Y]$. Before doing so we show how to complete the proof of the theorem. By assumption, there are integers $d_{e} \geq 0$ and sums of squares $s_{e} \in H^{0}\left(X, \mathcal{O}_{X}\left(2 d_{e}\right)\right)$, for $e \in\{0,1\}^{r}$, such that

$$
\frac{f h^{2}}{g^{2 t}}=\sum_{e \in\{0,1\}^{r}} \frac{s_{e}}{g^{2 d_{e}}} \cdot \frac{\left(h_{1} p_{1}\right)^{e_{1}} \cdots\left(h_{r} p_{r}\right)^{e_{r}}}{g^{2\left(a_{1} e_{1}+\cdots+a_{r} e_{r}\right)}}
$$


in $\mathbb{R}[Y]$. After multiplying with a sufficiently high even power of $g$ we get an identity

$$
f h^{2} g^{2 n}=\sum_{e \in\{0,1\}^{r}} t_{e} h_{1}^{e_{1}} \cdots h_{r}^{e_{r}}
$$

in $H^{0}\left(X, \mathcal{O}_{X}(2(t+n))\right)$, where $n \geq 0$ and $t_{e}$ is $s_{e}$ times an even power of $g$ and a product of some of the $p_{i}$.

Since the reduced section $h$ of $L^{\vee}(t)$ is weakly compatible with $K$, and since each summand $t_{e} h_{1}^{e_{1}} \cdots h_{r}^{e_{r}}$ on the right is nonnegative on $K$, it follows from Lemma 2.9(a) that $h$ divides $t_{e} h_{1}^{e_{1}} \cdots h_{r}^{e_{r}}$ for all $e$. Since no $h_{i}$ vanishes identically on any irreducible component of $\operatorname{supp}(h)$, it follows from Lemma 2.8 that $h$ divides $t_{e}$, for all e. Applying Lemma 2.9(b) we conclude that there are sums of squares $t_{e}^{\prime}$ with $t_{e}=h^{2} t_{e}^{\prime}$, for $e \in\{0,1\}^{r}$. We can therefore cancel $h^{2}$ from identity (2), see Lemma 2.7. and get

$$
f g^{2 n}=\sum_{e} t_{e}^{\prime} h_{1}^{e_{1}} \cdots h_{r}^{e_{r}}
$$

as desired.

It remains therefore to show that $\varphi$ lies in the preordering $T$ of $\mathbb{R}[Y]$ that is generated by $H_{1}, \ldots, H_{r}$. For this we use the local-global criterion of [14] Cor. 2.10. Note that this criterion does apply here since the preordering $T$ of $\mathbb{R}[Y]$ is archimedean, $K$ being compact (see, e.g., 9 Thm. 5.1.17). For any closed point $x$ of $Y$ we have to show $\varphi \in T_{x}$, where $T_{x}$ denotes the preordering generated by $H_{1}, \ldots, H_{r}$ in the local ring $\mathcal{O}_{X, x}$.

Let $u$ be a local generator of $L^{\vee}(t)$ at $x$. In $\mathcal{O}_{X, x}$ we have

$$
\varphi=\frac{f}{g^{2 t}} \cdot h^{2}=\frac{f u^{2}}{g^{2 t}} \cdot\left(\frac{h}{u}\right)^{2},
$$

and both factors $\varphi_{1}=\frac{f u^{2}}{g^{2 r}}$ and $\frac{h}{u}$ are in $\mathcal{O}_{X, x}$. It suffices to show $\varphi_{1} \in T_{x}$. By the hypotheses we have $\varphi_{1}>0$ on $\widetilde{K} \cap \operatorname{Sper}\left(\mathcal{O}_{X, x}\right)$, which is the basic closed subset of $\operatorname{Sper}\left(\mathcal{O}_{X, x}\right)$ associated with the preordering $T_{x}$. From [16] I. Prop. 2.1 it follows that $\varphi_{1} \in T_{x}$, and the proof is complete.

It is worthwile to isolate the unconstrained case (no $\left.h_{i}\right)$ :

Corollary 4.2. Let $X$ be a reduced projective $\mathbb{R}$-scheme without irreducible components of dimension one for which $X(\mathbb{R})$ is Zariski dense in $X$. Let L, $M$ be invertible sheaves on $X$, and let $f$ resp. $g$ be strictly positive global sections of $L^{\otimes 2}$ resp. $M^{\otimes 2}$. If $M$ is ample then $\mathrm{fg}^{n}$ is a sum of squares of global sections of $L \otimes M^{\otimes n}$ for all sufficiently large $n$.

Remark 4.3. Suppose that, in the situation of Theorem 4.1, we are in the particular case where $L=M^{\otimes k}$ for some $k \geq 0$. Then we get identity (11) for all sufficiently large $n \equiv k(\bmod 2)$ without the Zariski density hypothesis on $K$, and without any condition on the irreducible components of $X$. Indeed, we can directly apply Schmüdgen's Positivstellensatz [17] to the regular function $\varphi=\frac{f}{g^{k}}$ on $X_{g}$, which is strictly positive on $K$, and do not need to multiply with a factor $h$.

Remark 4.4. Theorem 4.1 remains true when $X$ is a nonsingular projective curve and $|K \cap O|=\infty$ for every oval $O$ of $X(\mathbb{R})$. Indeed, Theorem 3.5)(a) (plus Remark 3.7) is true in this case by Proposition 3.11. (We have actually not verified that $s$ in 3.5 (a) can also be chosen to be reduced, and leave it to the reader to show that this is true.) We do not know, however, if Theorem 4.1 holds true without the dimension restriction on the irreducible components of $X$, or without the condition that $K$ is Zariski dense in $X$. 
Corollary 4.5. Write $\mathrm{x}=\left(x_{0}, \ldots, x_{n}\right)$. Let $h_{1}, \ldots, h_{r} \in \mathbb{R}[\mathrm{x}]$ be homogeneous polynomials of even degree, and let

$$
S=\left\{\xi \in \mathbb{R}^{n+1}: h_{1}(\xi) \geq 0, \ldots, h_{r}(\xi) \geq 0\right\} .
$$

Assume there is $\xi \in \mathbb{R}^{n+1}$ with $h_{i}(\xi)>0$ for $i=1, \ldots, r$. If $f, g \in \mathbb{R}[\mathrm{x}]$ are homogeneous of (even) positive degree and strictly positive on $S \backslash\{(0, \ldots, 0)\}$, then $f g^{m}$ lies in the preordering generated by $h_{1}, \ldots, h_{r}$, for all sufficiently large $m \geq 0$.

Proof. That is, $f g^{m}$ satisfies an identity (11). This is just an affine reformulation of Theorem 4.1 in the case $X=\mathbb{P}^{n}$. Indeed, since $S$ has non-empty interior in $\mathbb{R}^{n+1}$, the corresponding projectivized semi-algebraic subset of $\mathbb{P}^{n}(\mathbb{R})$ is Zariski dense in $\mathbb{P}^{n}$.

Remark 4.6. In particular, if $f, g \in \mathbb{R}[\mathrm{x}]$ are positive definite forms of positive degree, then $f g^{m}$ is a sum of squares of forms for all $m \gg 0$. In this form the result was recently applied to time continuous dynamical systems by Ahmadi and Parrilo [1]. Using the result, the authors prove that, whenever a homogeneous polynomial Lyapunov function exists, there also exists one (of possibly higher degree) which is a sum of squares and whose negative derivative is also a sum of squares.

In [18, Stengle proved that there exist positive semidefinite forms $f$ in $\mathbb{R}[\mathrm{x}]$ of which no odd power $f^{2 m+1}$ is a sum of squares of forms. It follows directly from our result that this cannot happen when $f$ is strictly positive definite:

Corollary 4.7. Let $f \in \mathbb{R}[\mathrm{x}]$ be a strictly positive definite form. Then there exists an odd number $m \geq 1$ such that $f^{m}$ is a sum of squares of forms.

Remark 4.8. There is no such result for inhomogeneous strictly positive polynomials. For example, the polynomial $f=x^{3}+\left(x y^{2}-x^{2}-1\right)^{2}$ in $\mathbb{R}[x, y]$ is easily seen to be strictly positive on $\mathbb{R}^{2}$, but no odd power of $f$ is a sum of squares in $\mathbb{R}[x, y]$, according to Stengle [18].

4.9. As another concrete example we may apply the Positivstellensatz to products of projective spaces, or closed subvarieties thereof. For example, if $\mathrm{x}=\left(x_{0}, \ldots, x_{m}\right)$ and $\mathrm{y}=\left(y_{0}, \ldots, y_{n}\right)$ are coordinate tuples and $f, g \in \mathbb{R}[\mathrm{x}, \mathrm{y}]$ are positive definite bihomogeneous forms, where the bidegree $\left(d_{1}, d_{2}\right)$ of $g$ satisfies $d_{1}>0$ and $d_{2}>0$, then $f g^{N}$ is a sum of squares of bihomogeneous forms for all $N \gg 0$. Similarly for multihomogeneous forms.

From Theorem 4.1, it is possible to derive a necessary and sufficient condition, in terms of sums of squares, for a global section to be strictly positive. To keep the formulation simpler we restrict to the unconstrained case, however the statement holds with constraints as well. It also holds when $X$ is a nonsingular projective curve (Remark 4.4).

Corollary 4.10. Let $X$ be a reduced projective $\mathbb{R}$-scheme whose irreducible components have dimension $\neq 1$, let $L$ be an invertible sheaf on $X$, and let $f \in$ $H^{0}\left(X, L^{\otimes 2}\right)$. Then $f$ is strictly positive if and only if there is a very ample sheaf $M$ for which $L \otimes M$ is generated by global sections and for which the following condition holds for some basis $g_{1}, \ldots, g_{r}$ of $H^{0}(X, M)$ :

(*) For any $h \in H^{0}(X, L \otimes M)$ there exist a real number $\varepsilon>0$ and an integer $n \geq 0$ such that $g^{n}\left(g f-\varepsilon h^{2}\right)$ is a sum of squares of sections of $L \otimes M^{n+1}$, where $g:=g_{1}^{2}+\cdots+g_{r}^{2}$.

If $f>0$, condition $(*)$ is satisfied for any ample $M$ and any sequence $g_{1}, \ldots, g_{r}$ in $H^{0}(X, M)$ that generates $M$. 
Proof. First assume $f>0$, let $M$ be an ample invertible sheaf generated by global sections $g_{1}, \ldots, g_{r}$, and let $h \in H^{0}(X, L \otimes M)$. Writing $g:=g_{1}^{2}+\cdots+g_{r}^{2}$, there exists $\varepsilon>0$ such that $g f-\varepsilon h^{2}>0$. Indeed, this is clear locally on $X(\mathbb{R})$, and by compactness of $X(\mathbb{R})$ it is true globally. By Theorem 4.1 (or Corollary 4.2 in this case), there exists $n \geq 0$ such that $g^{n}\left(g f-\varepsilon h^{2}\right)$ is a sum of squares of sections of $L \otimes M^{n+1}$.

Conversely, assume that $M$ is ample and generated by global sections $g_{1}, \ldots, g_{r}$, that $L \otimes M$ is generated by global sections as well, and that $(*)$ holds for any $h \in H^{0}(X, L \otimes M)$. Given any point $\xi \in X(\mathbb{R})$, we choose $h$ such that $h(\xi) \neq 0$, and conclude $f(\xi)>0$.

In the case of nonsingular curves or surfaces, we even have a projective Nichtnegativstellensatz, due to the fact that psd $=$ sos holds in regular local rings of dimension $\leq 2$ :

Theorem 4.11. Let $X$ be a connected nonsingular projective curve or surface over $\mathbb{R}$ with $X(\mathbb{R}) \neq \varnothing$. Let $L, M$ be invertible sheaves on $X$, with $M$ ample, and let $f \in H^{0}\left(X, L^{\otimes 2}\right)$ and $g \in H^{0}\left(X, M^{\otimes 2}\right)$ be global sections with $f \geq 0$ and $g>0$ everywhere on $X(\mathbb{R})$. Then $f g^{N}$ is a sum of squares for all sufficiently large $N \geq 0$.

Proof. One proceeds as in the proof of Theorem 4.1 For $\operatorname{dim}(X)=2$ the proof can be copied word by word up to the last line. Due to the weaker assumption, we now only know $\varphi_{1} \geq 0$ (rather than $\varphi_{1}>0$ ) on $\operatorname{Sper}\left(\mathcal{O}_{X, x}\right)$. Replacing the reference to [16] 2.1 by [13 Thm. 4.8 resolves the matter. When $\operatorname{dim}(X)=1$, use Remark 3.10 instead of Theorem 3.5 to get the existence of $h$. The last step in the proof of 4.1 becomes elementary (an element in a discrete valuation ring $B$ containing $\frac{1}{2}$ that is a sum of squares in the field of fractions of $B$ is a sum of squares in $B$ ).

Remark 4.12. Various generalizations of Theorem 4.11 are possible that we won't explicate here in detail. For example, in the curve case we can allow ordinary real nodes on $X$ (use Monnier's theorem mentioned in 3.12, plus 13 Thm. 3.9). In the surface case we can allow nonreal singular points on $X$, provided their local rings are factorial (use [13] Cor. 4.9). We can also give constrained versions of the Nichtnegativstellensatz 4.11 provided the constraints $h_{i}$ satisfy local regularity conditions as in 14] Thm. 3.2, or more generally, as provided by the results of [16] I and II. For all these generalizations, it only matters that the preordering $T_{x}$ in $\mathcal{O}_{X, x}$ is saturated, for every closed point $x$ of $Y=X_{g}$ (see the end of the proof of Theorem 4.1).

\section{REFERENCES}

[1] A. A. Ahmadi, P. A. Parrilo: Converse results on existence of sum of squares Lyapunov functions. Preprint 2011.

[2] E. Becker: Valuations and real places in the theory of formally real fields. In: Géométrie Algébrique Réelle et Formes Quadratiques, Lect. Notes Math. 959, Springer, Berlin, 1982, pp. 1-40.

[3] J. Bochnak, M. Coste, M-F. Roy: Real Algebraic Geometry. Erg. Math. Grenzgeb. (3) 36, Springer, Berlin, 1998.

[4] R. Hartshorne: Algebraic Geometry. Grad. Texts Math. 52, Springer, New York, 1977.

[5] J.-P. Jouanolou: Théorèmes de Bertini et Applications. Progress in Mathematics 42, Birkhäuser, Boston, 1982.

[6] J.-Ph. Monnier: Divisors on real curves. Adv. Geom. 3, 339-360 (2003).

[7] J.-Ph. Monnier: On real generalized Jacobian varieties. J. Pure Applied Algebra 203, 252-274 (2005).

[8] D. Plaumann, C. Scheiderer: The ring of bounded polynomials on a semi-algebraic set. Trans. Am. Math. Soc. (to appear).

[9] A. Prestel, Ch. N. Delzell: Positive Polynomials. Monographs in Mathematics, Springer, Berlin, 2001. 
[10] B. Reznick: Uniform denominators in Hilbert's seventeenth problem. Math. Z. 220, 75-97 (1995).

[11] M. Roggero: Sui sistemi lineari e il gruppo delle classi di divisori di una varietà reale. Ann. Mat. Pura Appl. (4) 135, 349-362 (1984).

[12] C. Scheiderer: Sums of squares of regular functions on real algebraic varieties. Trans. Am. Math. Soc. 352, 1039-1069 (1999).

[13] C. Scheiderer: On sums of squares in local rings. J. reine angew. Math. 540, 205-227 (2001).

[14] C. Scheiderer: Sums of squares on real algebraic surfaces. Manuscr. math. 119, 395-410 (2006).

[15] C. Scheiderer: Positivity and sums of squares: A guide to recent results. In: Emerging Applications of Algebraic Geometry, IMA Vol. Math. Appl. 149, Springer, New York, 2009, pp. 271-324.

[16] C. Scheiderer: Weighted sums of squares in local rings and their completions I, II. Math. Z. 266, 1-19 (I) and 21-42 (II) (2010).

[17] K. Schmüdgen: The $K$-moment problem for compact semi-algebraic sets. Math. Ann. 289, 203-206 (1991).

[18] G. Stengle: Integral solution of Hilbert's seventeenth problem. Math. Ann. 246, 33-39 (1979).

Fachbereich Mathematik und Statistik, Universität Konstanz, 78457 Konstanz, GerMANY

E-mail address: claus.scheiderer@uni-konstanz.de 